\title{
Review
}

\section{Steroid regulation of programmed cell death during Drosophila development}

\author{
EH Baehrecke ${ }^{\star, 1}$ \\ 1 Center for Agricultural Biotechnology, University of Maryland Biotechnology \\ Institute, College Park, Maryland, MD 20742, USA \\ * Corresponding author: EH Baehrecke, Center for Agricultural Biotechnology, \\ University of Maryland Biotechnology Institute, College Park, Maryland, MD \\ 20742, USA. Tel: (301) 405-7525; Fax: (301) 314-9075 \\ E-mail: baehreck@umbi.umd.edu
}

Received 12.6.00; revised 3.7.00; accepted 4.7.00

Edited by S Kumar

\begin{abstract}
Steroid hormones play an important role in the regulation of numerous physiological responses, but the mechanisms that enable these systemic signals to trigger specific cell changes remain poorly characterized. Recent studies of Drosophila illustrate several important features of steroid-regulated programmed cell death. A single steroid hormone activates both cell differentiation and cell death in different tissues and at multiple stages during development. While several steroidregulated genes are required for cell execution, most of these genes function in both cell differentiation and cell death, and require more specific factors to kill cells. Genes that regulate apoptosis during Drosophila embryogenesis are induced by steroids in dying cells later in development. These apoptosis genes likely function downstream of hormone-induced factors to serve a more direct role in the death response. This article reviews the current knowledge of steroid signaling and the regulation of programmed cell death during development of Drosophila. Cell Death and Differentiation (2000) 7, 1057-1062.
\end{abstract}

Keywords: Steroid; ecdysone; development; metamorphosis; Drosophila

\section{Steroid hormones are important regulators of programmed cell death}

Steroid hormones serve a critical role in the maintenance of homeostasis. Steroids regulate metabolism, reproduction, and development in animals that are as different as insects and humans. During animal development, steroids trigger distinct responses including cell differentiation and programmed cell death. These hormones have been linked to numerous human health problems, and defects in hormonetriggered programmed cell death may result in the survival of tumor cells. ${ }^{1}$ In vertebrate organisms, steroids including androgens, estrogens, progesterone, and glucocorticoids regulate cell death. ${ }^{2-5}$ Glucocorticoid regulation of programmed lymphocyte death has served as a paradigm for steroid activation of apoptosis, and this response is dependent on glucocorticoid receptor function. ${ }^{6-10}$ In invertebrates, the steroid 20-hydroxyecdysone (ecdysone), and its receptor, have been implicated in the activation of programmed cell death during insect development. ${ }^{11-14}$

Steroids hormones appear to regulate programmed cell death by a variety of mechanisms. Most studies have reported that steroids serve as survival factors, and that hormone withdrawal results in the activation of programmed cell death. Examples of this mechanism include androgens in the prostate, ${ }^{15}$ and ecdysteroids in the insect nervous system. ${ }^{13}$ Alternatively, increases in steroids also activate programmed cell death. In Drosophila, increases in ecdysteroids trigger cell death in larval midguts and salivary glands. ${ }^{16}$ Glucocorticoid regulation of thymocyte cell death is complex and has been reported to be under both positive and negative control by this hormone, but recent in vivo studies indicate that a decrease in steroid titer regulates thymocyte apoptosis. ${ }^{17}$ While little is known about the steroid-regulated genes that control thymocyte and other vertebrate cell deaths, recent studies of Drosophila are providing insights into the genetic mechanisms underlying hormone-triggered programmed cell death.

\section{Steroid regulation of developmental changes at the onset of Drosophila metamorphosis}

The Drosophila life-cycle consists of embryonic, three larval instar, prepupal, pupal, and adult developmental stages. Pulses of the steroid hormone ecdysone punctuate each of these life stages, and regulate important transitions in development. ${ }^{18}$ During the onset of metamorphosis, fluctuations in ecdysone titer trigger dynamic cellular changes that are required to transform a larva into an adult (Figure 1). At the end of the third larval instar, an increase in ecdysone titer induces the formation of a prepupa. ${ }^{19-21}$ The ecdysone titer then drops to a low level in the mid-prepupal stage, ${ }^{20,21}$, and increases again $10-12 \mathrm{~h}$ following puparium formation. ${ }^{20,22}$ This pulse of ecdysone triggers future adult head eversion, which marks the beginning of pupation. ${ }^{20,22}$ The ecdysone titer then decreases at the onset of pupation before another large pulse of hormone occurs during pupal development. ${ }^{21}$

Metamorphosis of Drosophila involves the destruction of most of the larval tissues, and differentiation and morphogenesis of the tissues that form the adult fly (Figure 1). When the third instar larva turns into a prepupa, many tissues initiate metamorphic changes in 


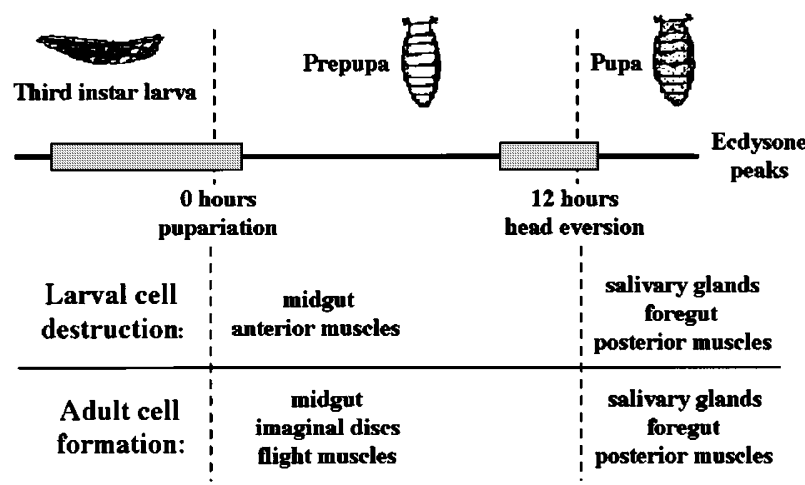

Figure 1 Fluctuations in the ecdysone titer during the onset of metamorphosis trigger stage- and cell-specific biological responses. Consecutive increases in ecdysone at the end of the third larval instar and $12 \mathrm{~h}$ later trigger the formation of a prepupa and pupa. These pulses of hormone also coincide with numerous tissue rearrangements including the death of larval cells and the formation of adult cells

synchrony with the rise in ecdysone titer. Imaginal discs undergo morphogenesis to form future adult appendages, and adult flight muscles appear in the anterior region of the prepupa. ${ }^{23,24}$ While these changes in adult structures occur, several tissues are destroyed including the anterior larval muscles and the larval midgut. ${ }^{23,24}$ Similarly, numerous cell and tissue changes are also induced when the ecdysone titer rises at the prepupal-to-pupal transition. The adult appendages deposit procuticle, ${ }^{25}$ the larval musculature completes its histolysis in the abdomen, the larval foregut epithelium is replaced by the adult foregut, and the larval salivary glands die while adult salivary glands initiate morphogenesis. ${ }^{23,24}$ While many changes associated with the transformation of a larva into an adult fly occur during the prepupal stage, additional details of adult formation are elaborated during the 3 days between pupation and adult eclosion.

Ecdysone triggers stage- and cell-specific changes, indicating that fluctuations in this systemic signal alone are not sufficient to determine the nature of the cellular response. Analyses of the mechanisms underlying ecdysone-regulated responses have been restricted to a limited number of tissues. Studies of ecdysone-triggered imaginal disc evagination have served as a useful model for morphogenesis. ${ }^{26,27}$ The nervous system, larval midgut, and larval salivary glands have been useful for studies of steroid regulation of cell death in Drosophila. ${ }^{13,16}$ While most studies of cell death have emphasized the common features of apoptosis, it should be noted that at least three types of programmed cell death occur during development of evolutionarily diverse organisms. ${ }^{28,29}$ During insect development, both apoptotic and autophagic cell death have been widely reported. ${ }^{28}$ Comparison of lymphocyte apoptosis and insect intersegmental muscle autophagy indicate that these physiological cell deaths occur by distinct mechanisms, ${ }^{30}$ but recent studies of Drosophila larval salivary glands suggest that these two types of cell death utilize some common mechanisms.

Drosophila salivary glands have been particularly useful as a model for steroid signaling, and possess several attributes making them an ideal system for studies of programmed cell death. Salivary gland cells die in a rapid and synchronous manner in response to the pulse of ecdysone that peaks $12 \mathrm{~h}$ following puparium formation. ${ }^{16}$ These cells exhibit dynamic changes in the tubulin and actin cytoskeleton, and accumulate acid phosphatase activity preceding their demise which appears to be mediated by lysosome-derived autophagic vacuoles. ${ }^{31,32}$ Markers of apoptosis including nuclear acridine orange staining and DNA fragmentation are detected by $14 \mathrm{~h}$ following puparium formation in salivary glands. ${ }^{16}$ Furthermore, salivary glands that are cultured in a physiologically elevated level of 20-hydroxyecdysone undergo programmed cell death. ${ }^{16}$

\section{Steroids signal by triggering a genetic regulatory hierarchy}

The mechanisms of steroid signaling have been extensively studied in Drosophila larval salivary glands because of the giant polytene chromosomes that form ecdysone-induced puffs reflecting a transcriptional regulatory hierarchy. Waves of chromosome puffs (decondensation of chromatin) accompany the late third instar larval and prepupal pulses of ecdysone. A series of elegant studies led to a model for genetic regulation of chromosome puffing. ${ }^{33-35}$ According to this model, the ecdysone receptor complex directly induces a small set of early puff genes, and the protein products of these genes then repress their own activity and induce a large set of secondary late response genes.

The isolation and characterization of the ecdysone receptor and ecdysone-regulated puff genes have provided substantial support for the model proposed based on chromosome puffing. ${ }^{36,37}$ The $E c R^{38}$ and $u s p^{39-41}$ genes both encode members of the nuclear hormone receptor family of proteins, and heterodimerize to form the ecdysone receptor. ${ }^{42,43}$ This receptor complex binds to DNA, and activates transcription of early puff genes, as early puffs and the genes encoded by these genetic loci are not properly induced in EcR and usp mutants. ${ }^{44,45}$ The characterization of the $B R-C, E 74$, and $E 75$ early puff genes provided further support of the puffing model for ecdysone signaling. ${ }^{46-48}$ These early puff genes are complicated and encode multiple isoforms of transcription factor proteins by alternative promoter usage and splicing. $B R-C$ encodes zinc finger proteins, E74 encodes members of the ETS family of DNA binding proteins, and E75 encodes nuclear hormone receptor family member zinc finger proteins. E74 and E75 proteins bind to both early and late puff chromosome loci. ${ }^{49,50}$ Late puff genes have not been extensively characterized, but the isolation of the $L 71$ late genes ${ }^{51,52}$ have been useful for testing the tenets of the steroid signaling model that was based on chromosome puffing. $B R-C$ and $E 74$ mutations impact transcription of late target genes. ${ }^{53}$ Furthermore, BR-C and E74 proteins bind to glue and $L 71$ gene regulatory elements, providing a direct link between these DNA binding proteins and the regulation of target gene transcription. ${ }^{54-56}$

The steroid regulatory hierarchy is activated by different pulses of ecdysone during development (Figure 2). The 


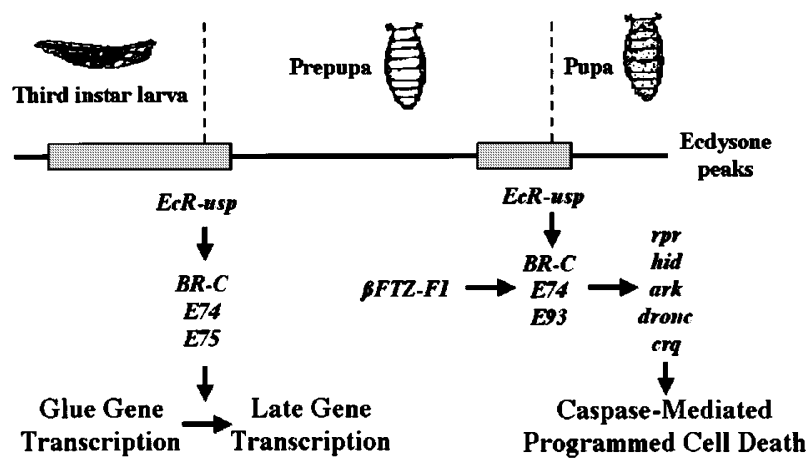

Figure 2 Steroid genetic regulatory hierarchy in Drosophila larval salivary glands. The rise of ecdysone at the end of the third laval instar triggers a change in salivary gland glue to late gene transcription, and this change is mediated by the ecdysone receptor (encoded by the EcR and usp genes) and the $B R-C, E 74$, and $E 75$ early puff genes. The subsequent increase in ecdysone titer at the end of prepupal development triggers caspase-mediated programmed cell death. This steroid-triggered cell death is regulated by the ecdysone receptor complex and $\beta F T Z-F 1$, which enable ecdysone induction of the $B R-C, E 74$, and $E 93$ genes. These regulatory factors are required for proper induction of the cell death genes $r p r$, hid, ark, dronc, and crq in salivary glands

increase in ecdysone titer at the end of the third larval instar regulates the transcription of glue and late genes in the salivary gland. $51,52,57,58$ The ecdysone titer then drops to a low level in midprepupae, enabling the induction of the nuclear hormone receptor $\beta F T Z-F 1{ }^{59} \beta F T Z-F 1$ serves as competence factor that enables the reinduction of the $B R-C$ and $E 74$ early genes, and the stage-specific induction of E93 by the pulse of ecdysone $12 \mathrm{~h}$ after puparium formation in salivary glands. ${ }^{59,60}$ While late puffs are observed at this stage of development, ${ }^{61}$ none of these late genes have been identified based on puffing. However, targets of the early genes that are induced at this stage have been identified. ${ }^{62,63}$

\section{Steroid-regulated genes function in programmed cell death}

Several ecdysone-regulated genes function in programmed cell death (Figure 2). The EcR, usp, $\beta F T Z-F 1, B R-C, E 74$, and E93 genes have been implicated in the regulation of programmed cell death in a variety of tissues including the midgut, salivary glands, and nervous system. ${ }^{13,14,45,60,62-64}$ $E c R$, usp, $\beta F T Z-F 1, B R-C$, and E74 are pleiotropic, however, and function in cell responses other than death including the proper formation of adult cells. ${ }^{44,60,65}$ Significantly, E93 appears to function more specifically in the destruction of larval tissues. ${ }^{63}$ E93 encodes a novel nuclear protein that is expressed in larval midgut and salivary gland cells immediately prior to ecdysone induction of their death. Furthermore, E93 mutants have defects in larval salivary gland cell destruction, and expression of E93 is sufficient to induce programmed cell death. If one reconsiders the steroid regulatory hierarchy in salivary glands, a cell death signaling hierarchy emerges (Figure 2). The ecdysone receptor complex and $\beta F T Z-F 1$ regulate $B R-C, E 74$, and $E 93$ transcription in larval salivary glands immediately prior to the initiation of cell destruction. ${ }^{59,60}$ E93 mutants possess decreased transcription of the $B R-C$ and $E 74$ genes, and each of these early genes impact the transcription of programmed cell death genes prior to the stage that larval salivary glands initiate destruction. ${ }^{62,63}$

\section{Apoptosis genes are regulated by steroid-induced genes prior to programmed cell death}

Drosophila possesses the programmed cell death pathway components that have been conserved in organisms as different as nematodes and humans. ${ }^{66}$ Caspases including DCP-1, Dredd, DrICE, Dronc, and Decay, ${ }^{67-72}$ the CED4/ APAF-1 homolog Ark, ${ }^{73-75}$ the CED-9/Bcl-2 family member Drob-1/Debcl/dBorg-1, ${ }^{76-78}$ and the inhibitors of apoptosis DIAP1 and DIAP2 ${ }^{79}$ have been identified. In addition, the novel rpr, hid, and grim cell death genes have been isolated and molecularly characterized. ${ }^{80-82}$

Several apoptosis genes have been implicated in ecdysone regulated programmed cell death (Figure 2). An increase in rpr and grim transcription foreshadows ecdysone induction of neuronal cell death, ${ }^{83}$ and mutations that remove these genes prevent these neurons from dying. ${ }^{84}$ In larval midguts and salivary glands, rpr and hid transcription increase prior to ecdysone-regulated programmed cell death. ${ }^{16}$ The core cell death machinery also appears to be involved in ecdysone-regulated programmed cell death. Inhibition of caspases by expression of the baculovirus inhibitor p35 blocks midgut and salivary gland cell death. ${ }^{16}$ In addition, ark and dronc transcription increase immediately prior to programmed cell death salivary glands. ${ }^{63}$

Ecdysone-regulated genes are required for proper transcription of apoptosis genes in salivary glands. $r p r$ transcription is directly regulated by the ecdysone receptor complex, but $B R-C$ function is also required for maximum levels of $r p r$ mRNA transcript in dying larval salivary glands. ${ }^{62}$ While mutations in the $E 74 A$ gene do not impact rpr transcription, both $B R-C$ and $E 74 A$ are required for proper transcription of hid in dying salivary glands. ${ }^{62}$ E93 mutants also impact the levels of several important apoptosis genes in salivary glands. ${ }^{63}$ Mutations in E93 result in decreased levels rpr, hid, ark, dronc, and crq RNA transcription. While E93 mutants do not impact the transcription of $E c R$ or $\beta F T Z-F 1$, these mutants do impact transcription of $B R-C$ and $E 74 A$. E93 protein binds to sites in the salivary gland polytene chromosomes that contain both steroid-regulated genes and programmed cell death genes. ${ }^{63}$ These data suggest that E93 regulates the apoptosis genes by either directly impacting their transcription, or indirectly by impacting early genes such as $B R-C$ and $E 74$ that in turn regulate cell death gene expression.

\section{Concluding remarks}

The regulation of programmed cell death plays a critical role during animal development by functioning in the destruction of unneeded cells and tissues. ${ }^{85,86}$ Proper implementation of a cell death response is also important for the removal of 
abnormal cells during development including tumor cells. ${ }^{1}$ Studies of Drosophila larval salivary glands have been emphasized in this review because of the utility of this tissue for studies of steroid signaling. However, steroids are only one of many developmental signals that activate programmed cell death in Drosophila and other organisms. ${ }^{87}$ Furthermore, many possible regulatory pathways could modulate cell death following the initial activation of death signaling. While cell death is regulated at the post-transcriptional level in Drosophila, ${ }^{88-90}$ emphasis has been placed on transcriptional control of cell death in this review, since steroid activity is mediated by nuclear receptor DNA binding proteins.

Larval tissues are destroyed by programmed cell death during Drosophila metamorphosis. The coordination of ecdysone induction of both cell death and cell differentiation during metamorphosis indicates that fluctuations in this systemic signal alone can't be responsible for the complexity of cell responses. Rather, expression of the proper combinations of regulatory proteins appears to be critical for the appropriate activation of genes that play a more direct role in the cell response. Some of these regulatory proteins, such as $E c R$, usp, $\beta F T Z-F 1, B R-C$, and $E 74$, regulate both cell differentiation and cell death, indicating that other more specific factors must specify the type of cell response to steroid. The E93 gene appears to specify ecdysone induction of cell death during metamorphosis, but it also appears to require other regulatory factors to properly activate the programmed cell death response. Many of the genes that function in the regulation of apoptosis during Drosophila embryogenesis are also involved in steroid activation of larval salivary gland cell death. Clearly, one of the most difficult challenges is to identify the novel components of the signaling pathways that lead to cell death, and integrate this knowledge into the development of the organism. The conservation of steroid signaling and apoptosis pathways in diverse organisms indicates that future genetic studies of Drosophila should lead to advances in understanding the mechanisms of programmed cell death and its regulation in higher organisms.

\section{Acknowledgements}

I thank the members of the Baehrecke laboratory for helpful discussions and comments on this manuscript. Work on this subject has been supported by NRICGP/USDA grant 9501913 and NIH grant GM59136.

\section{References}

1. Thompson CB (1995) Apoptosis in the pathogenesis and treatment of disease. Science 267: 1456-1462

2. Briehl MM and Miesfeld RL (1991) Isolation and characterization of transcripts induced by androgen withdrawal and apoptotic cell death in the rat ventral prostate. Mol. Endocrinol. 5: 1381-1388

3. Rotello RJ, Lieberman RC, Lepoff RB and Gerschenson LE (1992) Characterization of uterine epithelium apoptotic cell death kinetics and regulation by progesterone and RU 486. Am. J. Pathol. 140: 449-456

4. Tenniswood MP, Guenette RS, Lakins J, Mooibroek M, Wong P and Welsh J-E (1992) Active cell death in hormone-dependent tissues. Cancer Met. Rev. 11 $197-220$
5. Billig H, Furata I and Hsueh AJW (1993) Estrogens inhibit and androgens enhance ovarian granulosa cell apoptosis. Endocrinology 133: 2204-2212

6. Compton MM and Cidlowski JA (1986) Rapid in vivo effects of glucocorticoids on the integrity of rat lymphocyte genomic deoxyribonucleic acid. Endocrinology 118: $38-45$

7. Dieken ES and Miesfeld RL (1992) Transcriptional transactivation functions localized to the glucocorticoid receptor $\mathrm{N}$ terminus are necessary for steroid induction of lymphocyte apoptosis. Mol. Cell. Biol. 12: 589-597

8. Helmberg A, Auphan N, Caelles C and Karin M (1995) Glucocorticoid-induced apoptosis of human leukemic cells is caused by the repressive function of the glucocorticoid receptor. EMBO J. 14: 452-460

9. Cohen JJ and Duke RC (1984) Glucocorticoid activation of a calcium-dependent endonucleasein thymocyte nuclei leads to cell death. J. Immunol. 132: 38-42

10. Wyllie AH (1980) Glucocorticoid-induced thymocyte apoptosis is associated with endogenous endonuclease activation. Nature 284: 555-556

11. Lockshin RA and Williams CM (1964) Programmed cell death-II. Endocrine potentiation of the breakdown of the intersegmental muscles of silkmoths. J. Insect Physiol. 10: 643-649

12. Lockshin RA and Williams CM (1965) Programmed cell death-I. Cytology of degeneration in the intersegmental muscles of the pernyi silkmoth. J. Insect Physiol. 11: 123-133

13. Robinow S, Talbot WS, Hogness DS and Truman JW (1993) Programmed cell death in the Drosophila CNS is ecdysone-regulated and coupled with a specific ecdysone receptor isoform. Development 119: 1251-1259

14. Truman JW, Talbot WS, Fahrbach SE and Hogness DS (1994) Ecdysone receptor expression in the CNS correlates with stage-specific responses to ecdysteroids during Drosophila and Manduca development. Development 120: $219-234$

15. Isaacs JT (1984) Antagonistic effect of androgen on prostatic cell death. Prostate 5: $547-557$

16. Jiang C, Baehrecke EH and Thummel CT (1997) Steroid regulated programmed cell death during Drosophila metamorphosis. Development 124: 4673-4683

17. Vacchio MS, Lee JYM and Ashwell JD (1999) Thymus-derived glucocorticoids set the thresholds for thymocyte selection by inhibiting TCR-mediated thymocyte activation. J. Immunol. 163: 1327-1333

18. Riddiford LM (1993) Hormones and Drosophila development. In The development of Drosophila melanogaster, Bate M, Martinez Arias A, eds (Cold Spring Harbor: Cold Spring Harbor Laboratory Press) pp. 899-940

19. Richards G (1981) The radioimmune assay of ecdysteroid titres in Drosophila melanogaster. Mol. Cell. Endocrinol. 21: 181-197

20. Handler AM (1982) Ecdysteroid titres during pupal and adult development in Drosophila melanogaster. Dev. Biol. 93: 73-82

21. Pak MD and Gilbert LI (1987) A developmental analysis of ecdysteroids during the metamorphosis of Drosophila melanogaster. J. Liq. Chrom. 10: 2591-2611

22. Sliter TJ and Gilbert LI (1992) Developmental arrest and ecdysteroid deficiency resulting from mutations at the dre4locus of Drosophila. Genetics 130:555-568

23. Bodenstein D (1965) The postembryonic development of Drosophila. In Biology of Drosophila, Demerec M, ed (New York: Hafner Publishing Co.) pp. 275-367

24. Robertson CW (1936) The metamorphosis of Drosophila melanogaster, including an accurately timed account of the principal morphological changes. J. Morphol. 59: 351-399

25. Doctor J, Fristrom D and Fristrom JW (1985) The pupal cuticle of Drosophila: biphasic synthesis of pupal cuticle proteins in vivo and in vitro in response to 20hydroxyecdysone. J. Cell Biol. 101: 189-200

26. von Kalm L, Fristrom D and Fristrom J (1995) The making of a fly leg: a model for epithelial morphogenesis. BioEssays 17: 693-702

27. Fristrom D and Fristrom JW (1993) The metamorphic development of the adult epidermis. In The development of Drosophila melanogaster, Bate M, Martinez Arias A, eds (Cold Spring Harbor: Cold Spring Harbor Laboratory Press) pp. 843-897

28. Clarke PGH (1990) Developmental cell death: morphological diversity and multiple mechanisms. Anat. Embryol. 181: 195-213

29. Schweichel J-U and Merker H-J (1973) The morphology of various types of cell death in prenatal tissues. Teratology 7: 253-266

30. Schwartz LM, Smith SW, Jones MEE and Osborne BA (1993) Do all programmed cell deaths occur via apoptosis? Proc. Natl. Acad. Sci. USA 90: 980-984

31. Jochova J, Zakeri Z and Lockshin RA (1997) Rearrangement of the tubulin and actin cytoskeleton during programmed cell death in Drosophila salivary glands. Cell Death Differ. 4: 140-149 
32. von Gaudecker B and Schmale E-M (1974) Substrate-histochemical investigations and ultrahistochemical demonstrations of acid phosphatase in larval and prepupal salivary glands of Drosophila melanogasater. Cell Tissue Res. 155: 75-89

33. Ashburner M, Chihara C, Meltzer P and Richards G (1974) Temporal control of puffing activity in polytene chromosomes. Cold Spring Harbor Symp. Quant. Biol. 38: $655-662$

34. Becker HJ (1959) Die puffs der speicheldrüsenchromosomen von Drosophila melanogaster. I. Beobachtungen zur verhalten des puffmusters in normalstamm und bei zwei mutanten, giantund lethal-giant-larvae. Chromosoma 10:654-678

35. Clever U (1964) Actinomycin and puromycin: effects on sequential gene activation by ecdysone. Science 146: 794-795

36. Andres AJ and Thummel CS (1992) Hormones, puffs, and flies: the molecular control of metamorphosis by ecdysone. Trends Genet. 8: 132-138

37. Ashburner M (1990) Puffs, genes, and hormones revisited. Cell 61: 1-3

38. Koelle MR, Talbot WS, Segraves WA, Bender MT, Cherbas P and Hogness DS (1991) The DrosophilaEcR gene encodes an ecdysone receptor, a new member of the steroid receptor superfamily. Cell 67: 59-77

39. Henrich VC, Sliter TJ, Lubahn DB, MacIntyre A and Gilbert LI (1990) A steroid/ thyroid hormone receptor superfamily member in Drosophila melanogaster that shares extensive sequence similarity with a mammalian homologue. Nucleic Acids Res. 18: 4143-4148

40. Oro AE, McKeown M and Evans RM (1990) Relationship between the product of the Drosophila ultraspiraclelocus and vertebrate retinoid X receptor. Nature 347 : $298-301$

41. Shea MJ, King DL, Conboy MJ, Mariani BD and Kafatos FC (1990) Proteins that bind to Drosophila chorion cis-regulatory elements: a new $\mathrm{C}_{2} \mathrm{H}_{2}$ zinc finger protein and $\mathrm{a} \mathrm{C}_{2} \mathrm{C}_{2}$ steroid receptor-like component. Genes Dev. 4: 1128-1140

42. Thomas HE, Stunnenberg HG and Stewart AF (1993) Heterodimerization of the Drosophila ecdysone receptor with retinoid $\mathrm{X}$ receptor and ultraspiracle. Nature 362: $471-475$

43. Yao T-P, Segraves WA, Oro AE, McKeown M and Evans RM (1992) Drosophila ultraspiracle modulates ecdysone receptor function via heterodimer formation. Cell 71: 63-72

44. Bender M, Imam FB, TalbotWS, Ganetzky B and Hogness DS (1997) Drosophila ecdysone receptor mutations reveal functional differences among receptor isoforms. Cell 91: 777-788

45. Hall BL and Thummel CS (1998) The RXR homolog Ultraspiracle is an essential component of the Drosophila ecdysone receptor. Development 125: 4709 4717

46. Burtis KC, Thummel CS, Jones CW, Karim FD and Hogness DS (1990) The Drosophila $74 \mathrm{EF}$ early puff contains $E 74$, a complex ecdysone-inducible gene that encodes two ets-related proteins. Cell 61: 85-99

47. DiBello PR, Withers DA, Bayer CA, Fristrom JW and Guild GM (1991) The Drosophila Broad-Complex encodes a family of related proteins containing zinc fingers. Genetics 129: 385-397

48. Segraves WA and Hogness DS (1990) The E75 ecdysone-inducible gene responsible for the 75B early puff in Drosophila encodes two new members of the steroid receptor superfamily. Genes Dev. 4: 204-219

49. Urness LD and Thummel CS (1990) Molecular interactions within the ecdysone regulatory hierarchy: DNA binding properties of the Drosophila ecdysoneinducible E74A protein. Cell 63: 47-61

50. Hill RJ, Segraves WA, Choi D, Underwood PA and Macavoy E (1993) The reaction with polytene chromosomes of antibodies raised against Drosophila E75 protein. Insect Biochem. Mol. Biol. 23: 99-104

51. Restifo LL and Guild GM (1986) An ecdysone-responsive puff site in Drosophila contains a cluster of seven differentially regulated genes. J. Mol. Biol. 188:517528

52. Wright LG, Chen T, Thummel CS and Guild GM (1996) Molecular characterization of the 71E late puff in Drosophila melanogaster reveals a family of novel genes. J. Mol. Biol. 255: 387-400

53. Fletcher JC and Thummel CS (1995) The ecdysone-inducible Broad-Complex and $E 74$ early genes interact to regulate target gene transcription and Drosophila metamorphosis. Genetics 141: 1025-1035

54. Crossgrove K, Bayer CA, Fristrom JW and Guild GM (1996) The Drosophila Broad-Complex early gene directly regulates late gene transcription during the ecdysone-induced puffing cascade. Dev. Biol. 180: 745-758

55. Urness LD and Thummel CS (1995) Molecular analysis of a steroid-induced regulatory hierarchy: the Drosophila E74A protein directly regulates $L 71-6$ transcription. EMBO J. 14:6239-6246
56. von Kalm L, Crossgrove K, Von Seggern D, Guild GM and Beckendorf SK (1994) The Broad-Complex directly controls a tissue-specific response to the steroid hormone ecdysone at the onset of Drosophila metamorphosis. EMBO J. 13: 3505-3516

57. Crowley TE and Meyerowitz EM (1984) Steroid regulation of RNAs transcribed from the Drosophila 68C polytene chromosome puff. Dev. Biol. 102: 110-121

58. Hansson L and Lambertsson A (1989) Steroid regulation of glue protein genes in Drosophila melanogaster. Hereditas 110: 61-67

59. Woodard CT, Baehrecke EH and Thummel CS (1994) A molecular mechanism for the stage-specificity of the Drosophila prepupal genetic response to ecdysone. Cell 79: 607-615

60. Broadus J, McCabe JR, Endrizzi B, Thummel CS and Woodard CT (1999) The Drosophila $\beta$ FTZ-F1 orphan nuclear receptor provides competence for stagespecific responses to the steroid hormone ecdysone. Mol. Cell 3: 143-149

61. Ashburner M (1967) Patterns of puffing activity in the salivary gland chromosomes of Drosophila. I. Autosomal puffing patterns in a laboratory stock of Drosophila melanogaster. Chromosoma 21: 398-428

62. Jiang C, Lamblin A-FJ, Steller H and Thummel CT (2000) A steroid-triggered transcriptional hierarchy controls salivary gland cell death during Drosophila metamorphosis. Mol. Cell 5: 445-455

63. Lee C-Y, Wendel DP, Reid P, Lam G, Thummel CT and Baehrecke EH (2000) E93 directs steroid-triggered programmed cell death in Drosophila. Mol. Cell: 6 : $433-443$

64. Restifo LL and White K (1992) Mutations in a steroid hormone-regulated gene disrupt the metamorphosis of internal tissues in Drosophila: salivary glands, muscle, and gut. Wilhelm Roux's Arch. Dev. Biol. 201: 221-234

65. Fletcher JC, Burtis KC, Hogness DS and Thummel CS (1995) The Drosophila E74 gene is required for metamorphosis and plays a role in the polytene chromosome puffing response to ecdysone. Development 121: 1455-1465

66. Abrams JM (1999) An emerging blueprint for apoptosis in Drosophila. Trends Cell Biol. 9: $435-440$

67. Chen P, Rodriguez A, Erskine R, Thach T and Abrams JM (1998) Dredd, a novel effector of the apoptosis activators Reaper, Grim, and Hid in Drosophila. Dev. Biol. 201: 202-216

68. Dorstyn L, Colussi PA, Quinn LM, Richardson H and Kumar S (1999) DRONC, an ecdysone-inducible Drosophila caspase. Proc. Natl. Acad. Sci. USA 96: 4307 4312

69. Dorstyn L, Read SH, Quinn LM, Richardson H and Kumar S (1999) DECAY, a novel Drosophila caspase related to mammalian caspase-3 and caspase-7. J. Biol. Chem. 274: 30778-30783

70. Fraser AG and Evan GI (1997) Identification of a Drosophila melanogaster ICE CED-3-relatedprotease, drICE. EMBO J. 16(10): 2805-2813

71. Inohara N, Koseki T, Hu Y, Chen S and Nunez G (1997) CLARP, a death effector domain-containing protein interacts with caspase-8 and regulates apoptosis. Proc. Natl. Acad. Sci. USA 94: 10717-10722

72. Song Z, McCall K and Steller H (1997) DCP-1, a Drosophila cell death protease essential for development. Science 275: 536-540

73. Kanuka H, Sawamoto K, Inohara N, Matsuno K, Okano H and Miura M (1999) Control of the cell death pathway by Dapaf-1, a DrosophilaApaf-1/CED-4-related caspase activator. Mol. Cell 4: 757-769

74. Rodriguez A, Oliver H, Zou H, Chen P, Wang X and Abrams JM (1999) Dark is a Drosophila homologue of Apaf-1/CED-4 and functions in an evolutionarily conserved death pathway. Nat. Cell Biol. 1: 272-279

75. Zhou L, Song Z, Tittel J and Steller H (1999) HAC-1, a Drosophila homolog of APAF-1 and CED-4, functions in developmental and radiation-induced apoptosis. Mol. Cell 4: 745-755

76. Baker Brachmann C, Jassim OW, Wachsmuth BD and Cagan RL (2000) The Drosophila Bcl-2 family member dBorg-1 functions in the apototic response to UV-irradiation. Curr. Biol. 10: 547-550

77. Colussi PA, Quinn LM, Huang DCS, Coombe M, Read SH, Richardson H and Kumar S (2000) Debcl, a proapoptotic Bcl-2 homologue, is a component of the Drosophila melanogaster cell death machinery. J. Cell Biol. 148: 703-714

78. Igaki T, Kanuka H, Inohara N, Sawamoto K, Nunez G, Okano H and Miura M (2000) Drob-1, a Drosophilamember of the Bcl-2/CED-9 family that promotes cell death. Proc. Natl. Acad. Sci. USA 97: 662-667

79. Hay BA, Wassarman DA and Rubin GM (1995) Drosophila homologs of baculovirus inhibitor of apoptosis proteins function to block cell death. Cell 83 : $1253-1262$ 
80. Chen P, Nordstrom W, Gish B and Abrams JM (1996) grim, a novel cell death gene in Drosophila. Genes Dev. 10: 1773-1782

81. Grether ME, Abrams JM, Agapite J, White K and Steller H (1995) The head involution defective gene of Drosophila melanogasterfunctions in programmed cell death. Genes Dev. 9: 1694-1708

82. White K, Grether ME, Abrams JM, Young L, Farrell Kand Steller H (1994) Genetic control of programmed cell death in Drosophila. Science 264: 677-683

83. Robinow S, Draizen TA and Truman JW (1997) Genes that induce apoptosis: transcriptional regulation in identified, doomed neurons of the Drosophila CNS. Dev. Biol. 190: 206-213

84. Draizen TA, Ewer J and Robinow S (1999) Genetic and hormonal regulation of the death of peptidergic neurons in the Drosophila central nervous system. J. Neurobiol. 38: 455-465

85. Jacobson MD, Weil M and Raff MC (1997) Programmed cell death in animal development. Cell 88: 347-354
86. Vaux DL and Korsmeyer SJ (1999) Cell death in development. Cell 96: 245-254

87. McCall K and Steller H (1997) Facing death in the fly: genetic analysis of apoptosis in Drosophila. Trends Genet. 13: 222-226

88. Goyal L, McCall K, Agapite J, Hartwieg E and Steller H (2000) Induction of apoptosis by Drosophila reaper, hid and grim through inhibition of IAP function. EMBO J. 19: 589-597

89. Lisi S, Mazzon I and White K (2000) Diverse domains of THREAD/DIAP1 are required to inhibit apoptosis induced by REAPER and HID in Drosophila. Genetics 154: 669-678

90. Wang SL, Hawkins CJ, Yoo SJ, Müller H-AJ and Hay BA (1999) The Drosophila caspase inhibitor DIAP1 is essential for cell survival and is negatively regulated by HID. Cell $98: 453-463$ 